J. Clin. Chem. Clin. Biochem.

Vol. 17, 1979, pp. 105-110

\title{
Nachweis einer typenspezifischen Mikroheterogenität des humanen Thyroxin-bindenden Globulins durch isoelektrische Fokussierung
}

\section{Von W. Petek}

Institut für Medizinische Biochemie (Vorstand Prof. Dr. A. Holasek) der Universität Graz

(Eingegangen am 2. Juni/11. September 1978)

\begin{abstract}
Zusammenfassung: Es wird eine rasch durchführbare Methode zur Isolierung des Thyroxin-bindenden Globulins aus nur $150 \mathrm{ml}$ Plasma beschrieben. Wesentlich war die Präparation einer hochaktiven Thyroxin-Sepharose, die ausschließlich kovalent gebundenes $L$-Thyroxin enthielt, und die Elution des Proteins unter spezifisch hydrophoben Bedingungen. Durch isoelektrische Fokussierung von 15 individuellen Präparationen des Thyroxin-bindenden Globulins gelang es nach densitometrischer Auswertung der Polyacrylamidgele, eine unterschiedliche Verteilung der drei darstellbaren Proteinbanden nachzuweisen. Innerhalb der untersuchten Probanden konnten drei Verteilungstypen nachgewiesen werden. Die Ergebnisse dieser Untersuchung weisen darauf hin, daß die Typen individualspezifisch sind, während geschlechtsspezifische Unterschiede nicht nachweisbar waren.
\end{abstract}

\section{Evidence of type-specific microheterogeneity of thyroxine-binding globulin by isoelectric focusing}

Summary: A rapid method for the isolation of the thyroxine-binding globulin from only $150 \mathrm{ml}$ plasma is described. The preparation of a high-activity thyroxine-sepharose is essential; this contained $L$-thyroxine bound exclusively by covalent linkage. Furthermore, the elution of the protein was performed under specifically hydrophobic conditions. Using isoelectric focusing on 15 individual preparations of thyroxine-binding globulin, and densitometric analysis of the polyacrylamide gels, it was possible to show three protein bands in different proportions according to the individual. Three distribution-types were found in the tested volunteers. The results of this investigation show that these types are individual-specific, whereas there are no apparent sex-specific differences.

\section{Einleitung}

Das Thyroxin-bindende Globulin ${ }^{1}$ ist das wichtigste Transportprotein für die Schilddrüsenhormone $L-3,5,3^{\prime}$ Triiodthyronin und $L$-Thyroxin ${ }^{2}$. Seine Serumkonzentration, $10-20 \mathrm{mg} / 1$, ist ein bedeutender Parameter bei der in vịtro Diagnostik von Schilddrüsenerkrankungen $(1,2,3)$. Es wurde in verschiedenen Laboratorien aus Serum oder Plasma isoliert und teilweise charakterisiert (4-9). Nilsson èt al. (10) postulierten, daß das TBG aus vier identischen Untereinheiten aufgebaut sei, eine Behauptung, die kurze Żeit später von Gershengorn et al. (11) wịderlegt wuŕde. Vor längerer Zeit konnte aber an TBG-Präparationen durch isoelektrische Fokussierung nachgewiesen werden, $d a ̣ ß$ das Protein eine polymorphe Mikroheterogenität aufweist. Dies wurde erstmals von Marshall et al. (12) mitgeteilt, wobei 4
Subfraktionen angegeben wurden. Von Horn et al. (13) konnten jedoch an reinsten TBG-Präparationen aus Mischserum nur 3 Subfraktionen festgestellt werden. Es war daher naheliegend zu untersuchen, ob nicht durch geringfügige Unterschiede in den physiko-chemischen Eigenschaften individueller TBG-Präparationen differente Verteilungsmuster von TBG-Subfraktionen nachgewiesen werden können.

\section{Material und Methoden \\ Probenmaterial}

Das Acidum-Citricum-Dextrose-Plasma ${ }^{3}$ stammte von klinisch gesunden Spendern und wurde vom Department für Blutgruppenserologie der Univ. Klinik für Chirurgie, Graz, zur Verfügung gestellt. Das Mischserum wurde aus verschiedenen klinischen Laboratorien bezogen.

\footnotetext{
1) Thyroxin-bindendes Globulin

2) $L$-Thyroxin

3) Acidum-Citricum-Dextrose-Plasma
}

TBG
$T_{4}$
ACD-Plasma

4) $L$-Thyroxin-Sepharose
5) 8-Anilinonaphthalinsulfon Ammoniumsalz
T -Sephyrose ANS

0340-076X/79/0105-0110\$02.00

(c) by Walter de Gruyter \& Co. · Berlin - New York 


\section{Reagenzien}

Sepharose 4 B, ConcanavalinA-Sepharose und Sephadex G 25 fine stammten von Pharmacia. $\mathrm{Na}^{125} \mathrm{I}(3,7 \mathrm{TBq} / \mathrm{l})$ und das ${ }^{125} \mathrm{I}$ Thyroxin $(1,85 \mathrm{GBq} / \mathrm{l})$ waren Produkte von Radiochemical Centre, Amersham.

Der mikrogränuläre Anionenaustauscher DiethylaminoethylCellulose, DE-32, stammte von der Firma Whatman. Das $\alpha$-Methylmannosid war von SIGMA und die Agarose ein Produkt von Bio-Rad. Sämtliche Reagenzien für die Diskelektrophorese und die analytische Elektrofokussierung waren von Serva, nur das Ampholine pH 3,5-5,0 stammte von LKB (Bromma, Schweden). Die Lactoperoxidase (EC 1.11.1.7) wurde von BoehringerMannheim bezogen. Die verschiedenen Antihumanseren stammten von Behring, das Thiazinrot $\mathbf{R}$ war von Fluka. Das Antiserum „Kaninchen Immunglobuline gegen das Thyroxin-bindende Globulin" 10-K 40 stammte von Dakopatts (Medac, Hamburg). Alle anderen Chemikalien waren von analytischer Reinheit und wurden von Merck Darmstadt bezogen.

Geräte

Zur Molekulargewichtsbestimmung wurde eine analytische Beckman Ultrazentrifuge Modell E verwendet. Die Auswertung der Polyacrylamidgele erfolgte mit einem Densitometer der Firma Vernon (Paris). Der Gammazähler Typ BF 1015 war ein Produkt von Berthold. Die RC-5 Hochgeschwindigkeitszentrifuge stammte von Sorvall, (Newton, Mass., USA). Die Einrichtung für die analytische Fokussierung stammte von LKB.

Präparation der $L$-Thyroxin-Sepharose 4

Die für die biospezifische Adsorption des TBG erforderliche $\mathrm{T}_{4}$ Sepharose wurde durch kovalente Bindung von $\mathrm{T}_{4}$ an die Gelmatrix hergestellt. $100 \mathrm{ml}$ feuchte Sepharose $4 \mathrm{~B}$ wurden nach der Bromcyanaktivierungsmethode von Cuatrecasas (14) nach einer Modifikation von Pensky et al. (4) mit $130 \mu \mathrm{mol} \mathrm{T} \mathrm{T}_{4}$ umgesetzt. Die Ausbeute bei der Kupplung des $\mathrm{T}_{4}$ an die Sepharose wurde durch Einsatz von ${ }^{125} \mathrm{I}$-Thyroxin ermittelt. Sie betrug unter optima!en Bedingungen $50-60 \%$ der eingesetzten $\mathrm{T}_{4-}$ Menge entsprechend einer Beladung des Gels mit 0,65-0,78 $\mathrm{mmol} / 1 \mathrm{~T}_{4}$. Trotz vieler Vorversuche und vor allem Änderung der eingesetzten $T_{4}$-Menge wiesen die $T_{4}$-Sepharosen aber eine unterschiedliche Qualität auf. Um unspezifisch an der $\mathrm{T}_{4}$-Sepharose haftendes $\mathrm{T}_{4} \mathrm{zu}$ entfernen, wurde die Methode (4) insofern modifiziert, als nach dem Abblocken noch aktiver Gruppen mit Ethanolamin die frische $\mathrm{T}_{4}$-Sepharose zuerst für $10 \mathrm{~min}$ in $0,1 \mathrm{~mol} / 1 \mathrm{NaOH}$ inkubiert wurde.

Hydrophobe Natur der TBG Bindung an die $\mathrm{T}_{4}$-Sepharose

21 Mischserum wurden mit $80 \mathrm{ml} \mathrm{T}_{4}$-Sepharose und 7,4 MBq ${ }_{125} \mathrm{I}$-TBG $2 \mathrm{~h}$ bei Raumtemperatur inkubiert. $\mathrm{Di}_{2}: \mathrm{T}_{4}$-Sepharose wurde hernach auf einer Glasfritte $G 2$ portionsweise mit 31 $10 \mathrm{mmol} / 1$ Tris/ $\mathrm{HCl}$ Puffer, $0,5 \mathrm{~mol} / 1 \mathrm{NaCl}$, pH 8,0 weitgehend proteinfrei gewaschen, in eine Säule $(2,5 \times 30 \mathrm{~cm})$ gefüllt und mit 2.x $500 \mathrm{ml}$ gleichem Puffer eluiert. Hierauf wurde die $\mathrm{T}_{4}$-Sepharose mit $2 \times 500 \mathrm{ml} 10 \mathrm{mmol} / 1 \mathrm{Tris} / \mathrm{HCl}$ Puffer $\mathrm{pH} 8,0$ gewaschen und anschließend das TBG mit $3 \times 500 \mathrm{ml} 10 \mathrm{mmol} / \mathrm{l}$ Tris/ $\mathrm{HCl}$ Puffer, $0,16 \mathrm{mmol} / 18$ Anilinonaphthalinsulfonsäure(1) Ammoniumsalz ${ }^{5}$, pH 8,0 abgelöst. Die Ausbeute an TBG bei der Adsorption wurde aus der eingesetzten Aktivität und deren Wiederfindung im Serum nach der Inkubation berechnet. Die Verluste bei den Waschvorgängen durch Verminderung der Salzkonzentration des Puffers und die Prozentsätze an Aktivität, die durch ANS-haltigen Puffer eluiert werden konnten, sind aus Tabelle $1 \mathrm{zu}$ ersehen.

\section{Präparation des TBG aus ACD-Plasma}

$150 \mathrm{ml}$ Plasma wurden $20 \mathrm{~min}$ bei $13700 \mathrm{~g}$ in einer Sorvall- $\cdot$ Hochgeschwindigkeitszentrifuge RC-5 zentrifugiert. Die Adsorption des TBG aus dem Plasma erfolgte anschließend durch $10 \mathrm{ml} \mathrm{T}_{4}$-Sepharose in Plastiksäulen $(8,5 \times 1,8 \mathrm{~cm})$ mit einer Durchlaufgeschwindigkeit von $1,0 \mathrm{ml} / \mathrm{min}$. Unspezifisch gebundene Plasmaproteine wurden durch extensives Waschen mit $500 \mathrm{ml} 0,1 \mathrm{~mol} / 1$ Tris/ $\mathrm{HCl}$ Puffer $\mathrm{pH} 8,0$ aus der Säule entfernt. Die Elution des TBG gemeinsam mit anderen $\mathrm{T}_{4}$-bindenden Proteinen erfolgte hierauf mit $120 \mathrm{ml} \mathrm{0,1} \mathrm{mol} / 1$ Tris/HCl Puffer $0,16 \mu \mathrm{mol} / 1 \mathrm{~T}_{4}, \mathrm{pH} 8,0$, direkt auf einer mit $1 \mathrm{ml}$ ConA-Se- pharose gefüllte Plastiksäule $(10,0 \times 0,8 \mathrm{~cm})$, wobei Albumin und Präalbumin quantitativ entfernt werden konnten. An-

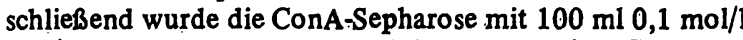
Tris/HCl pH 8,0 gewaschen und das TBG sowie $\gamma$-Globuline mit

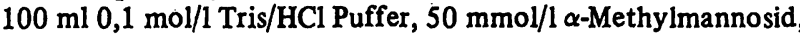
pH 8,0 eluiert und sofort gegen einen $50 \mathrm{mmol} / 1 \mathrm{Tris} / \mathrm{HCl}$ Puffer $\mathrm{pH} 8,0$ dialysiert. Die endgültige Reinigung erfolgte über eine DE-32 $(1 \mathrm{ml})$ in Plastiksäulen $(10,0 \times 0,8 \mathrm{~cm})$, wobei durch Waschen der DE-32 mit $50 \mathrm{ml} 50 \mathrm{mmol} / 1 \mathrm{Tris} / \mathrm{HCl}$ Puffer, $50 \mathrm{mmol} / 1 \mathrm{NaCl}, \mathrm{pH} 8,0$ diè $\gamma$-Globuline entfernt werden konnten. Das TBG wurde durch $10 \mathrm{ml} 50 \mathrm{mmol} / 1 \mathrm{Tris} / \mathrm{HCl}$ Puffer, $80 \mathrm{mmol} / 1 \mathrm{NaCl}$, pH 8,0 abgelöst und das Volumen des Eluates durch Druckdialyse auf $2 \mathrm{ml}$ eingeengt.

\section{Maximale $\mathrm{T}_{\mathbf{4}}$-Bindungskapazität}

$20 \mu \mathrm{g} \mathrm{TBG}$ in $100 \mu \mathrm{l} 0,1 \mathrm{~mol} / \mathrm{l}$ Tris/HCl Puffer, $0,8 \mu \mathrm{mol} / 1 \mathrm{~T}_{4}$, $\mathrm{pH} 8,0$ wurden $30 \mathrm{~min}$ bei Raumtemperatur inkubiert. Hierauf wurde das gebundene $T_{4}$ in Plastiksäulen $(10,0 \times 0,5 \mathrm{~cm})$, die mit $3 \mathrm{ml}$ Sephadex G 25 fine gefüllt waren, mit einem $0,1 \mathrm{~mol} / 1$ Tris/HCl Puffer $\mathrm{pH} 8,0$ vom freien $\mathrm{T}_{4}$ abgetrennt. Das an TBG gebundene $T_{4}$ wurde durch nasse Veraschung als $T_{4}$-Iod $(15,16)$ bestimmt. Die quantitative TBG-Bestimmung erfolgte mittels Elektroimmunodiffusion (17) unter gleichen Bedingungen, wie bei Kranz et al. (18) angegeben, wobei ein selbst hergestelltes Anti-TBG-Serum von Kaninchen eingesetzt wurde. Als Standard diente ein mit TBG aufgestocktes Normalserum, dessen TBGKonzentration (73,5 mg/l) von Dr. Bradwell, Birmingham, England, bestimmt worden war.

\section{Iod Markierung des TBG}

Das zur Iodmarkierung verwendete reinste TBG wurde, wie oben beschrieben, aus Mischplasma isoliert. Es wurde sowoh nach der Chloramin-T Methode (19) als auch nach der Lactoperoxidase-Methode (20) mit einer Ausbeute von 20-30\% iodiert. Wegen der größeren Verluste an Bindungsfähigkeit für $\mathrm{T}_{4}$ durch Oxidation des Proteins bei der Chloramin-T Methode (21) wurde der schonenderen Iodierung mit Lactoperoxidase der Vorzug gegeben.

\section{Elektrophoretische Methoden}

Zur Reinheitsüberprüfung der TBG-Präparation wurde die analytische Diskelektrophorese nach Weber \& Osborne (22) verwendet. Die immunelektrophoretische Untersuchung der TBGRohpräparationen wurden in $20 \mathrm{~g} / \mathrm{l}$ Agarose in einem $20 \mathrm{mmol} / \mathrm{l}$ Barbitalpuffer pH 8,2, Ionenstärke $\mu=0,025$, durchgeführt. Die Laufzeit bei $5 \mathrm{~mA}$ pro Objektträger betrug $1,5 \mathrm{~h}$.

Molekulargewichtsbestimmung des TBG

Das Molekulargewicht des TBG wurde mit einer analytischen Ultrazentrifuge (Beckman Modell E) nach der SedimentationsGleichgewichts-Methode (23) bestimmt. Dazu wurde eine TBGPräparation aus Mischserum verwendet, die nach der gleichen Methode, wie bei Plasma angegeben, isoliert worden war. Die Proteinkonzentration betrug $0,5 \mathrm{~g} / \mathrm{l}(\rho=1,0055)$. Als $\overline{\mathrm{V}}$ wurde 0,734 angenommen. Die Laufzeit betrug $24 \mathrm{~h}$, die Rotordrehzahl 30000 Umdrehungen/min und die Rotortemperatur $\mathrm{T}$ $=298,0 \mathrm{~K}$.

\section{Isoelektrische Fokussierung der TBG-Präparationen}

Die analytische isoelektrische Fokussierung wurde mit einigen Abänderungen, die Vorbereitung der Färbelösung zur Gänze, nach Malik \& Berrie (24) durchgeführt.

Lösung A: $0,56 \mathrm{~mol} / \mathrm{i}$ Acrylamid und $52 \mathrm{mmol} / 1 \mathrm{~N}, \mathrm{~N}^{\prime}$ Methylenbisacrylamid

Lösung B: $16 \mathrm{mmol} / 1$ Riboflavin

Lösung C: $1,32 \dot{\mathrm{mmol}} / 1 \mathrm{~N}, \mathrm{~N}, \mathrm{~N}^{\prime}, \mathrm{N}^{\prime}$ Tetramethylethylendiamin Lösung D: $40 \mathrm{~g} / 1$ Ampholine $\mathrm{pH}$ 3,5-5,0

Je $380 \mu \mathrm{l}$ der Lösungen $A, B, C$ und $65 \mu$ D wurden vorgemischt und $760 \mu \mathrm{l}$ der wäßrigen TBG-Lösung $(71,0-97,5 \mathrm{mg} / \mathrm{l}$ TBG) hinzugefügt. Das Gemisch wurde in Glasröhrchen $(8,0 \times 0,5 \mathrm{~cm})$ gefüllt und hierauf $1 \mathrm{~h}$ bei $355 \mathrm{~nm}$ photopolymerisiert. Als Anodenflüssigkeit diente $20 \mathrm{mmol} / 1$ o-Phosphorsäure und kathodisch wurde $67 \mathrm{mmol} / 1$ Triethanolamin verwendet. Bei einer konstanten Spannung von $200 \mathrm{~V}$ wurde $10 \mathrm{~h}$ bei $4^{\circ} \mathrm{C}$ 
fokussiert. Zur Fixierung der Proteinbanden und zur Entfernung des Ampholines wurden die Gele $24 \mathrm{~h}$ in $0,76 \mathrm{~mol} / 1$ Trichloressigsäure eingebracht. Die Gele wurden für $24 \mathrm{~h}$ in der wegen Interferenz mit dem Ampholine speziell aufbereiteten Farbe Coomassie brilliant blue G 250 (24) inkubiert und anschließend in destilliertem Wasser aufbewahrt.

Statistische Methoden

Das Verhalten der durch isoelektrische Fokussierung darstellbaren drei Subfraktionen $F_{1}-F_{3}$ innerhalb der drei beschriebenen TBG-Verteilungstypen wurden mittels des Duncan-Tests untersucht (25).

\section{Ergebnisse}

Präparation des TBG aus ACD-Plasma

In den aus ACD-Plasma mit $\mathrm{T}_{4}$ als Effektor gewonnenen TBG-Präparationen konnten in den Eluaten nach der $\mathrm{T}_{4}$ Sepharose immunochemisch neben dem TBG noch Präalbumin, Albumin und $\boldsymbol{\gamma}$-Globuline nachgewiesen werden (Abb. 1). Nach der ConA-Sepharose waren als Verunreinigung noch $\boldsymbol{\gamma}$-Globuline zu finden, während nach der DE-32 immunochemisch und diskelektrophoretisch, wie aus Abbildung 2 ersichtlich, nur noch TBG in der Präparation vorhanden war. Diskelektrophoretisch waren die Präparationen bei drei verschiedenen Gelkonzentrationen und auch in harnstoffhaltigen Gelen einheitlich. Nur an der Grenze zwischen Sammelgel und Trenngel kam bei einzelnen Präparationen eine schwach anfärbbare Bande zur Darstellung. Trotz der extrem niedrigen physiologischen TBG-Konzentration von 10-20 $\mathrm{mg} / 1$ konnten davon $20-25 \%$ in reinster Form aus dem Plasma isoliert werden.

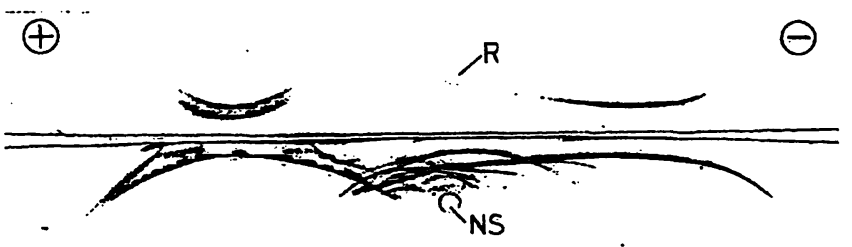

Abb. 1. Immunelektrophorese einer mit $T_{4}$ als Effektor abgelösten TBG-Rohpräparation $(R)$ und eines Normalserums (NS). Die Trénnung erfolgte in $20 \mathrm{~g} / 1$ Agarose in einem $20 \mathrm{mmol} / 1$ Barbitalpuffer pH 8,2, lonenstärke 0,025 ( $3 \mathrm{ml}$ pro Objektträger), durch $1,5 \mathrm{~h}$ bei $5 \mathrm{~mA}$ konst. Stromfluß. Entwickelt wurde mit einem polyvalenten Anti-Humanserum und die Färbung erfolgte mit Thiazinrot $R$. In der TBG-Rohpräparation ( $R$ ) siñ Präalbumin, Albumin und IgG nachweisbar.

\section{Hydrophobe Natur der TBG-Bindung}

Nach zweistündiger Inkubation der $\mathrm{T}_{4}$-Sepharose im Mischserum, welches eine durchschnittliche Osmolarität vön 0,29 osmol/1 besitzt, waren $80 \%$ der eingesetzten Aktivität gebunden. Das Verhalten des gebundeneñ Anteils des ${ }^{125}$ I-TBG während des Waschens der $\mathrm{T}_{4}$-Sepharose mit den beiden Puffern sowie die Ausbeute bei der Elution mit dem ANS-haltigen Puffer ist aus der Tabelle 1 ersichtlich. $5,2 \%$ der an der $\mathrm{T}_{4}$-Sepharose haftenden Aktivität konnten nur durch $0,1 \mathrm{~mol} / \mathrm{l} \mathrm{NaOH}$ entfernt werden. Die hydrophobe Natur der Bindung des TBG an die $\mathrm{T}_{4}$-Sepharose ist aus dem Verhalten beim Waschen

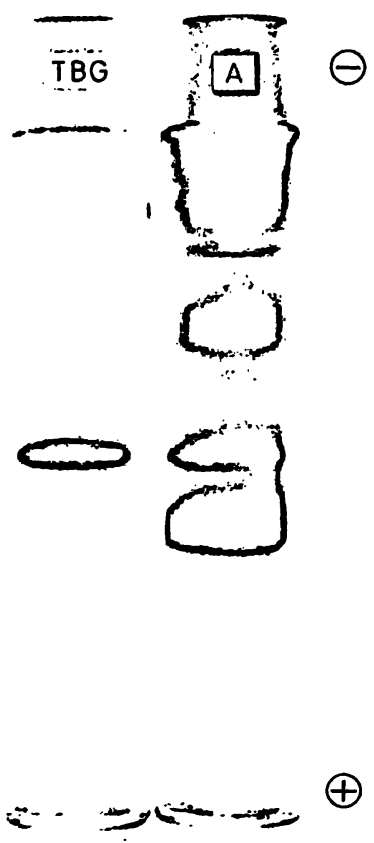

Abb. 2. Diskelektrophorese einer mit $10 \mathrm{mmol} / 1$ Tris/HCl Puffer, $0,16 \mathrm{mmol} / 1$ ANS, $\mathrm{pH}$ 8,0 gewonnenen TBG-Rohpräparation (A) und von $60 \mu \mathrm{g}$ reinstem TBG nach Ionenaustauschchromatographie mittels DE-32 (TBG). Die Färbung der Gele erfolgte mit $10 \mathrm{~g} / 1$ Amidoschwarz $10 \mathrm{~B}$ in $1,16 \mathrm{~mol} / 1$ Essigsäure.

durch Erniedrigung der Salzkonzentration des Puffers und der Elution durch ANS-haltigen Puffer zu ersehen. Die mit ANS gewonnenen TBG-Präparationen waren aber noch mit zumindest 11 anderen Plasmaproteinen verunreinigt (Abb. 3). Bei den weiteren Experimenten
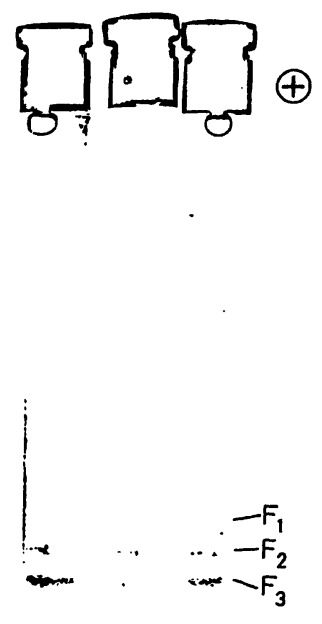

Abb. 3. Reinste TBG-Präparationen wurden in Polyacrylamidgelen, pH Gradient 3,5-5,0, durch Elektrofokussierung während $10 \mathrm{~h}$ bei $200 \mathrm{~V}$ konst., in drei Subfraktionen $\left(F_{1}-F_{3}\right)$ aufgetrennt. Die Proteinfraktionen wurden durch $0,76 \mathrm{~mol} / 1$ Trichloressigsäure fixiert und mit vorgereinigtem Coomassie brilliant blue G $\mathbf{2 5 0}$ gefärbt. 
wurde daher nur noch mit einer Puffermolarität von $0,1 \mathrm{~mol} / 1$ gearbeitet und als Effektor $\mathrm{T}_{4}$ eingesetzt.

Molekulargewicht und maximale $\mathrm{T}_{4}$-Bindungskapazität des TBG

Die Auswertung der Molekulargewichtsbestimmung nach Yphantis (23) ergab fur das TBG ein Molekulargewicht von 52800 Dalton. An mehreren TBG-Präparationen aus Mischserum und -plasma konnte eine maximale Beladung des Proteins mit $\mathrm{T}_{4}$ von $14,2 \mu \mathrm{g} / \mathrm{mg}$ TBG erhalten werden. Aus diesen beiden Daten folgt, daß $0,96 \mathrm{~mol} \mathrm{~T}_{4}$ pro $\mathrm{mol} \mathrm{TBG} \mathrm{gebunden} \mathrm{wurden} \mathrm{und}$ das TBG durch die hier beschriebene Präparationsmethode keine gravierenden Strukturänderungen erfahren haben kann.

\section{Subtypen des TBG}

Das TBG konnte durch isoelektrische Fokussierung in drei Banden unterschiedlich färbbarer Intensität aufgetrennt werden (Abb. 3). Wie aus Abbildung 4 ersichtlich, ergaben mit einem monospezifischen Anti-TBGSerum alle drei Fraktionen eine immunochemisch einheitliche Reaktion. Die Präzipitationsbanden zeigen Fusion und das Muster vollständiger immunochemischer Identität. Ein weiterer Beweis dafür, daß es sich bei den drei Banden nur um TBG handelt, konnte dadurch erbracht werden, daß mit einem Serum eines Patienten mit genetisch bedingtem Fehlen des TBG keine immunochemische Reaktion erhalten werden konnte. Der isoelektrische Punkt der drei Proteine wurde nach Ausschneiden der Fraktionen aus den Gelen in den wäßrigen Eluaten ermittelt. Die anodisch gelegene Fraktion wies einen $\mathrm{pH}$ Wert von $4,15\left(\mathrm{~F}_{1}\right)$, die mittlere einen $\mathrm{pH}$ von 4,30 $\left(\mathrm{F}_{2}\right)$ und die am weitesten kathodisch gelegene einen $\mathrm{pH}$ von $4,45\left(\mathrm{~F}_{3}\right)$ auf. Nach densitometrischer Auswertung der gefärbten Gele ließen sich aus der Relation des Proteingehaltes der Subfraktionen zueinander eindeutig 3 Typen feststellen (Abb. 5). Von den 15 Probanden zeigten 8 den höchsten $F_{2}$ Anteil und wurden als Typ I bezeichnet, während $\mathrm{F}_{3}$ beim Typ II dominierte. Beim Typ III war der Anteil von $\mathrm{F}_{2}: \mathrm{F}_{3}$ etwa gleich groß (Tab. 2). Der $F_{1}$ Anteil war im DuncanTest immer signifikant am niedrigsten. Innerhalb der Relativverteilung der drei Subfraktionen $\mathrm{F}_{1}-\mathrm{F}_{3}$ wiesen $F_{1}: F_{2}$ und $F_{1}: F_{3}$ bei allen drei Typen und $F_{2}: F_{3}$ $2^{\circ}$

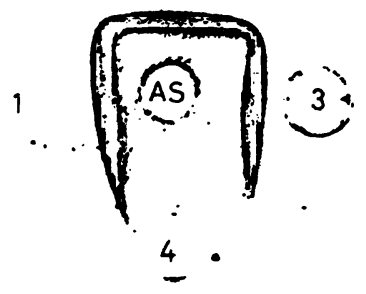

Abb. 4. Die Subfraktionen $F_{1}, F_{2}$ und $F_{3}$ wurden nach analytischer Fokussierung durch Elution aus den Gelen gewonnen. 1-3 Material mit dem isoelektrischen Punkt: 4,15, 4,30 bzw. 4,45; 4: Serum eines Patienten mit genetisch bedingtem Fehlen des TBG. AS: monospezifisches Anti-TBG-Serum von Kaninchen.
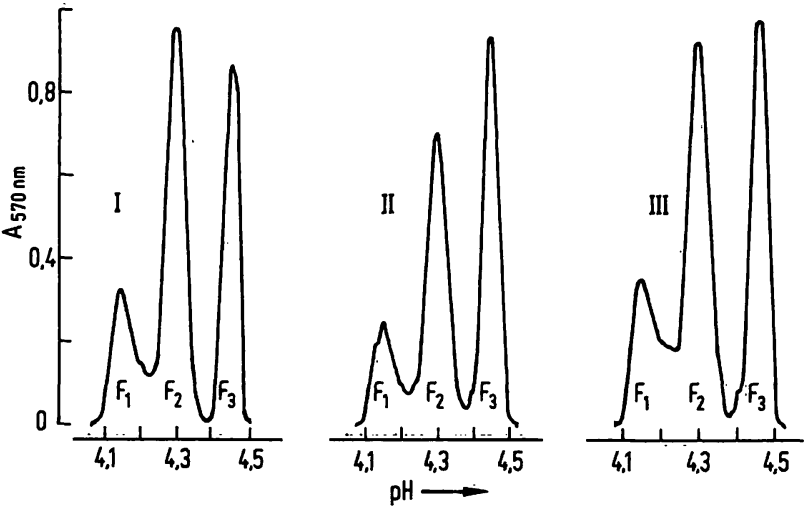

Abb. 5. Densitogramme von in Polyacrylamidgelen isoelektrisch fokussierten TBG-Präparationen. Unter gleichen Fokussierungsbedingungen wie in Abbildung 3 gelangten nach densitometrischer Auswertung der Gele bei $570 \mathrm{~nm}$ in jeder Präparation 3 Subfraktionen $\left(\bar{F}_{1}-\dot{F}_{3}\right)$ zur Darstellung. Innerhalb der 15 untersuchten Präparationen wurden 3 Verteilungstypen (Typ I-III) festgestellt. Die prozentuellen Anteile von $F_{1}: F_{2}$ und $F_{1}: F_{3}$ wiesen bei allen drei Typen im Duncan-Test ein $p<0,01$ und von $\mathrm{F}_{2}: \mathrm{F}_{3}$ beim Typ I und Typ II noch ein $\mathrm{p}<0,05$ auf, während beim Typ III kein signifikanter Unterschied bestand.

mit Ausnahme vom Typ III einen signifikanten Unterschied auf. Mit dem zahlenmäßig beschränktèn Material konnte ein Verhältnis der Typen I : II : IIII von $8: 5: 2$ erhoben werden. Geschlechtsspezifische Unterschiede waren nicht erkennbar.

Tab. 1. Einfluß von Puffermolarität, Natriumchloridkonzentration und ANS auf die Elution des ${ }^{125}$ I-TBG von der $T_{4}$-Sepharose.

\begin{tabular}{|c|c|c|c|c|c|c|}
\hline \multirow[b]{2}{*}{ Schritt } & \multirow[b]{2}{*}{$\begin{array}{l}\text { Volumen } \\
\text { (ml) }\end{array}$} & \multicolumn{5}{|l|}{ Puffer } \\
\hline & & $\begin{array}{l}\text { Tris/HCl } \\
(\mathrm{mmol} / \mathrm{l})\end{array}$ & $\mathrm{pH} \cdot$ & $\begin{array}{l}\mathrm{NaCl} \\
(\mathrm{mol} / \mathrm{l})\end{array}$ & $\begin{array}{l}\text { ANS } \\
\text { (mmol/l) }\end{array}$ & $\begin{array}{l}\text { Aktivität im Eluat } \\
(\%)\end{array}$ \\
\hline $\begin{array}{l}1 \\
2 \\
3 \\
4 \\
5 \\
6 \\
7\end{array}$ & $\begin{array}{l}500 \\
500 \\
500 \\
500 \\
500 \\
500 \\
500\end{array}$ & $\begin{array}{l}10 \\
10 \\
10 \\
10 \\
10 \\
10 \\
10\end{array}$ & $\begin{array}{l}8,0 \\
8,0 \\
8,0 \\
8,0 \\
8,0 \\
8,0 \\
8,0\end{array}$ & $\begin{array}{l}0,5 \\
0,5 \\
\end{array}$ & $\begin{array}{l}0,16 \\
0,16 \\
0,16\end{array}$ & $\begin{array}{r}2,2 \\
1,1 \\
33,2 \\
14,5 \\
29,5 \\
7,5 \\
6,7 .\end{array}$ \\
\hline
\end{tabular}


Tab. 2. Typenspezifische Mikroheterogenität des TBG.

\begin{tabular}{|c|c|c|c|c|}
\hline $\begin{array}{l}\text { Proband } \\
\text { Alter/ } \\
\text { Geschlecht }\end{array}$ & $\begin{array}{l}\text { TBG } \\
\text { Präparation } \\
\text { (mg/l) }\end{array}$ & $\begin{array}{l}\mathrm{F}_{1} \\
\mathrm{pH} 4,15 \\
(\mathrm{mg} / \mathrm{l})\end{array}$ & $\begin{array}{l}\mathrm{F}_{2} \\
\mathrm{pH} 4,30 \\
(\mathrm{mg} / \mathrm{l})\end{array}$ & $\begin{array}{l}\mathrm{F}_{3} \\
\mathrm{pH} 4,45 \\
(\mathrm{mg} / \mathrm{l})\end{array}$ \\
\hline \multicolumn{5}{|l|}{ Typ 1} \\
\hline $\begin{array}{l}488 \\
238 \\
258 \\
198 \\
37 \% \\
318 \\
248 \\
298\end{array}$ & $\begin{array}{l}86,9 \\
71,9 \\
75,0 \\
86,9 \\
72,8 \\
82,1 \\
74,9 \\
71,0\end{array}$ & $\begin{array}{l}16,9 \\
14,2 \\
15,8 \\
16,8 \\
16,6 \\
19,7 \\
16,0 \\
10,8\end{array}$ & $\begin{array}{l}38,1 \\
29,5 \\
32,3 \\
38,2 \\
29,0 \\
35,5 \\
30,3 \\
31,5\end{array}$ & $\begin{array}{l}31,9 \\
28,2 \\
26,9 \\
31,9 \\
27,2 \\
26,9 \\
28,6 \\
28,7\end{array}$ \\
\hline \multicolumn{5}{|l|}{ Typ II } \\
\hline $\begin{array}{l}19 \circ \\
20 \% \\
35 \% \\
27 \% \\
368\end{array}$ & $\begin{array}{l}83,0 \\
79,7 \\
87,7 \\
76,5 \\
88,2\end{array}$ & $\begin{array}{r}11,3 \\
8,7 \\
11,2 \\
13,4 \\
10,0\end{array}$ & $\begin{array}{l}28,8 \\
29,7 \\
32,8 \\
28,9 \\
36,1\end{array}$ & $\begin{array}{l}42,9 \\
41,3 \\
43,7 \\
34,2 \\
42,1\end{array}$ \\
\hline \multicolumn{5}{|l|}{ Typ III } \\
\hline $\begin{array}{l}23 \delta \\
19 \delta\end{array}$ & $\begin{array}{l}86,4 \\
97,5\end{array}$ & $\begin{array}{l}13,8 \\
13,9\end{array}$ & $\begin{array}{l}36,3 \\
41,6\end{array}$ & $\begin{array}{l}36,3 \\
42,0\end{array}$ \\
\hline
\end{tabular}

\section{Diskussion}

Negative Einflüsse auf die Bindungsqualität der $\mathrm{T}_{4^{-}}$ Sepharose resultieren aus der schlechten Löslichkeit des $\mathrm{T}_{4}$ bei pH 8,6. Bereits bei der Kupplung an die bromcyanaktivierte Sepharose bleibt ein Teil des eingesetzten $\mathrm{T}_{4}$ unspezifisch am $\mathrm{Gel}$ haften und fuhrt bei der Adsorption des TBG aus dem Plasma zu unterschiedlich großen Verlusten. Durch Waschen der frisch bereiteten $\mathrm{T}_{4}$-Sepharose mit $0,1 \mathrm{~mol} / 1 \mathrm{NaOH}$ wird das gesamte nicht kovalent gebundene $\mathrm{T}_{4}$ vom $\mathrm{Gel}$ entfernt und ermöglicht es, Ausbeuten bis zu $80 \%$ zu erreichen.

Der Effektor, 2 mmol/1 KOH (4), erzeugt in der Säule durch Pufferwirkung der Proteine nur einen $\mathrm{pH}$-Wert von 9,2 (11). Wie eigene Vorversuche zeigten, konnte durch einen 0,1 mol/1 Tris/HCl Puffer $\mathrm{pH}$ 9,2 nur eine geringe Menge TBG von der $\mathrm{T}_{4}$-Sepharose eluiert werden. Zum anderen wirkt sich aber die extrem niedere Molarität der Kalilauge nachteilig auf die Reinheit der TBGRohpräparationen aus. Neben dem TBG konnten in diesen Eluaten immunochemisch noch 11 weitere Serumproteine nachgewiesen werden. Die Elution des TBG bei relativ hoher Puffermolarität mit dem spezifischen Effektor $\overline{\mathrm{T}}_{4}$ lieferte hingegen wesentlich reinere TBGRohpräparationen.

In Übereinstimmung mit Horn et al. (13) konnten in den TBG.Präparationen aus Serum und Plasma drei Subfraktionen festgestellt werden. Gegensätzlich verhalten sich dazu die Ergebnisse von Marshall et al. (12), die zumindest 4 Subfraktionen postulieren. Eịne Erklärung für diese divergierenden Ergebnisse von diesen beiden Arbeitsgruppen ist durch den Einsatz unterschiedlicher Ëffektoren gegeben. Bei Verwendung von Kalilauge oder
ANS-haltigen Puffern als Effektor konnte, wie in Vorversuchen festgestellt, immer eine größere Menge an $\alpha_{1}$-Antitrypsin im Eluat nachgewiesen werden. Mehrere physiko-chemische Eigenschaften dieses Proteins (26), wie Molekulargewicht, isoelektrischer Punkt, und die Zusammensetzung des Kohlenhydratanteils besitzen eine große Ähnlichkeit mit denen des TBG (11).

Bei Verwendung dieser beiden unspezifischen Effektoren ist eine vollständige Abtrennung des $\alpha_{1}$-Antitrypsins aus den TBG-Präparationen auch mittels der hier beschriebenen Methode nie gelungen.

Eine maximale Ausbeute an TBG aus einem geringen Ausgangsvolumen konnte nur durch stufenweise Elution erreicht werden. Ebenso dauerte die Summe aller Präparationsschritte nicht länger als zwei Tage, womit die Gefahr einer enzymatisch bedingten Degradation des Proteins weitestgehend vermindert war. Die maximale Bindungsfähigkeit des TBG für $\mathrm{T}_{4}$ ist mit von anderen gefundenen Ergebnissen ( 9, 13, 27) gut vergleichbar. Sie beweist, daß die Tertiärstruktur der Bindungsstelle für das $\mathrm{T}_{4}$ durch die vorausgegangene Präparation nicht verändert wurde. Das festgestellte Molekulargewicht von 52800 Dalton befindet sich in dem in der Literatur mitgeteilten Bereich $(5,11,28)$.

Reinste TBG-Präparationen, die nach der hier beschriebenen Methode hergestellt wurden, ergaben nach wiederholter Immunisierung von Kaninchen ein monospezifisches Antiserum. Trotzdem war es erforderlich, die aus individuellen TBG-Präparationen durch isoelektrische Fokussierung erhaltenen Subfraktionen immunochemisch zu charakterisieren. Mit polyvalenten und monospezifischen Antiseren gegen menschliche Serumproteine (Behring) konnte kein Resultat erhalten werden. Nur mit dem Antiserum ,Kaninchen Immunglobuline gegen das Thyroxin-bindende Globulin" 10-K 40 konnte mittels zweidimensionaler Immunodiffusion und immunelektrophoretisch jeweils ein Präzipitat erhalten werden.

Innerhalb der 15 individuellen TBG-Präparationen konnten durch analyitische Elektrofokussierung unter Annahme gleicher Farbbindung innerhalb der drei Subfraktionen eindeutig drei Verteilungstypen (Typ I-III) festgestellt werden. Vorläufig bleibt ungewiß, ob die hier beschriebene Mikroheterogenität des TBG bereits bei der Proteinsynthese entsteht oder ob es sich um eine postsynthetische Mikroheterogenität (29) handelt. Die relativ großen $\mathrm{pH}$-Differenzen zwischen den einzelnen isoelektrischen Punkten der drei Subfraktionen könnten auch als Ausdruck einer großen Molekuillabilität des TBG angesehen werden. Damit kann nicht gänzlich ausgeschlossen werden, daß die drei Subfraktionen während der Präparation entstehen und eher auf einen postsynthetischen Ursprung hinweisen. Das konstante Auftreten von nur drei Subfraktionen innerhalb der untersuchten Probanden ist aber damit schwer vereinbar, da bei einer postsynthetischen Mikroheterogenität eine 
weitaus größere Anzahl von Subfraktionen zu erwarten ist. Aus den vorliegenden Ergebnissen können keine endgültigen Schlüsse über die Ursache für das Auftreten dieser Mikroheterogenität gezogen werden. Da es sich beim TBG um ein Glykoprotein handelt, war auch zu vermuten, daß die einzelnen Subfraktionen sich durch einen unterschiedlichen Neuraminsäuregehalt voneinander unterscheiden. Von Marshall et al. (12) wurde aber nachgewiesen, daß die vollständige Abspaltung der endständigen Neuraminsäuren vom Kohlenhydratanteil des Proteins zwar eine Verschiebung der isoelektrischen Punkte aller Subfraktionen in Richtung geringerer Acidität bewirkt, das Bandenmuster aber als solches erhalten bleibt. Eine Artefarktbildung ist deswegen auszuschliessen, da die wiederholte Präparation vom selben Probanden das gleiche Verteilungsmuster der Subfraktionen ergab. Unwahrscheinlich ist auch, daß die drei Bänden durch eine unterschiedliche $T_{4}$-Bindung hervorgerufen werden, da mit $\mathrm{T}_{4}$ oder ANS gewonnene Präparationen, unabhängig von der Verunreinigung mit $\alpha_{1}$-Antitrypsin

\section{Literatur}

1. Pickardt, C. R., Bauer, M., Horn, K., Kubiczek, Th. \& Scriba, P. C. (1977), Internist 18, 538-543.

2. Burr, W. A., Ramsden, D. B., Evans, S. E., Hogan, T. \& Hoffenberg, R. (1977), Br. Med. J. 1, 485-488.

3. Hesch, R. D., Gatz, J., Pape, J., Schmidt, E. \& von zur Mühlen, A. (1976), Eur. J. Clin. Invest. 6, 139-145.

4. Pensky, J. \& Marshall, J. S. (1969), Arch. Biochem. Biophys. 135, 304-310.

5. Marshall, J. S. \& Pensky, J. (1969), J. Clin. Invest. 48, 508-515.

6. Tata, J. R. (1961), Clin. Chim. Acta 6, 819-832.

7. Giorgio, N. A. J. \& Tabachnik, M. (1968), J. Biol. Chem. 243, 2247-2259.

8. Kågedal, B. \& Källberg, M. (1977), Clin. Chim. Acta 78, $103-111$.

9. Sterling, K., Hamada, S., Takemura, Y., Brenner, M. A., Newman, E. S. \& Inada, M. (1971), J. Clin. Invest. 50, 1758-1771.

10. Nilsson, S. F. \& Peterson, P. A. (1975), J. Biol. Chem. 250, 8543-8553.

11. Gershengorn, M. C., Cheng, S. Y.., Lippoldt, R. E., Lord, R. S. \& Robbins, J. (1977), J. Biol. Chem, 252, 8713-8718.

12. Marshall, J. S., Pensky, J. \& Williams, St. (1973), Arch. Biochem. Biophys. 156, 456-462.

13. Horn, K., Kubiczek, Th., Pickardt, C. R. \& Scriba, P. C. (1977), Klin. Wochenschr. 55, 881-894.

14. Cuatrecasas, P. (1970), J. Biol. Chem. 245, 3059-3065. bei den letzteren, immer das gleiche Verhältnis der Subfraktionen aufwiesen.

Um endgültig zu klären, ob diese Subfraktionen des TBG genetisch bedingt sind, werden derzeit einige Familienuntersuchungen durchgeführt. Weiters wird versucht, größere Mengen an den einzelnen Subfraktionen zu isolieren, um eine physiko-chemische Charakterisierung durchführen zu können.

\section{Danksagung}

Besonderer Dank gilt Frau OA. Dr. Ilse Teubl, Leiter des Departments für Bluttransfusion und Blutgruppenserologie an der Chirurgischen Universitätsklinik Graz, für die zur Verfügung gestellten Plașmaproben, sowie Herrn Dr. R. A. Bradwell, Department of Medicine, University of Birmingham, für die quantitative Analyșe des in der Arbeit verwendeten TBGStandards. Diese Arbeit wurde außerdem vom Fonds zur Förderung der wissenschaftlichen Forschung durch Bereitstellung der analytischen Ultrazzentrifuge Beckman Modell E unterstützt.
15. Knapp, G. \& Spitzy, H. (1970), Clin. Chim. Acta 30, 119128.

16. Knapp, G. \& Leopold, H. (1974), Anal. Chem. 46, 719-723.

17. Laurell, C. B., (1972), Scand. J. Clin. Lab. Invest. 29, Suppl. $124,1-136$.

18. Kranz, Th., Trautwein, A. \& Sieber, A. (1974), diese Z. 12, 124-127.

19. Greenwood, F. C., Hunter, W. M. \& Glover, J. S. (1963), Biochem. J. 89, 114-123.

20. Thorell, J. I. \& Johannsson, B. G: (1971), Biochim. Biophys. Acta 251, 363-369.

21. Diel, F., Schneider, E. \& Quabbe, H. J. (1977), diese Z. 15, $669=677$.

22. Weber, K. \& Osborne, M. (1969), J. Biol. Chem. 244, 4406-4412.

23. Yphantiss, D. A. (1964), Biochemistry 3, 297-317.

24. Mali, N. \& Berrie, A. (1972), Anal. Biochem. 49, 173-176.

25. Duncan, D. B. (1955), Biometrics. 11, 1-42.

26. Chan, C. K., Luby, J. \& Wu, Y. C. (1973), FEBS Lett. 35, 79-82.

27. Cavalieri, R. R., McMahon, F. A. \& Castle, J. N. (1975), J. Clin. Invest. 56, 79-87.

28. Korcek, L. \& Tabachnik, M. (1974), Biochim. Biophys. Acta 371, 323-336.

29. Bours, J. (1976), in Isoelectric focusing (Catsimpoolas, N. Hrisg.) S. 225-228, Academic Press, Inc., New York.

Dr. W. Petek

Institut für Medizinische Biochemie der Universität Graz

Harrachgasse 21/III

A-8010 Graz/Austria 